\section{Evaluation of Broccoli and Cauliflower Germplasm for Resistance to Race 2 of Peronospora parasitica}

\author{
Claude E. Thomas ${ }^{1}$ and E.L. Jourdain ${ }^{2}$ \\ U.S. Department of Agriculture, Agricultural Research Service, U.S. \\ Vegetable Laboratory, Charleston, SC 29414
}

Additional index words. Brassica oleracea var. botrytis, B. oleracea var. italica, downy mildew, disease resistance, foliar disease

\begin{abstract}
Evaluations for resistance against race 2 of downy mildew, incited by Peronospora parasitica, were conducted on 240 U.S. Plant Introductions (PI) classified as Brassica oleracea var. botrytis (consists of both broccoli and cauliflower types). Plants were inoculated at the two-expanded leaf stage with $5.0 \times 10^{3}$ sporangia per $\mathrm{ml}$. Inoculated plants were incubated in a dark 16C dew chamber for $24 \mathrm{hr}$ and were then placed in a 22C growth chamber with a 12-hr photoperiod. On the 7th day after inoculation, plants were returned to the dew chamber for $30 \mathrm{hr}$ and ratings for downy mildew reaction phenotypes were made at 9 days postinoculation on a $0-9$ scale of increasing disease severity. A disease index (DI) was calculated for each entry. Based on the DI, no PI entries were highly resistant. PI entries 181860, 188562, 204765, 204768,204772,204773, 204775,204779,241612, 264656,291567,373906, and 462225 were moderately resistant. (DI of 3.1-5).
\end{abstract}

Downy mildew of broccoli (Brassica oleracea var. italica L.), cauliflower (B. oleracea var. botrytis L.), and other crucifers is incited by Peronospora parasitica Pers. ex Fr. The disease is serious in the cooler parts of Europe, the United States, India, Russia, South America, and Australia (Sherf and Macnab, 1986). The high humidity, warm days, and cool nights of the southeastern United States result in severe damage from this disease (Walker and Williams, 1977). Biological races of $P$. parasitica occur on the Cruciferae and three races have been recognized on B. oleracea (Natti et al., 1967; M.H. Dickson, personal communication).

Received for publication 31 Mar. 1989. Mention of a trade name or a proprietary product does not constitute a guarantee or warranty of the product by the USDA and does not imply approval of it to the exclusion of other products that also may be suitable. The cost of publishing this paper was defrayed in part by the payment of page charges. Under postal regulations, this paper therefore must be hereby marked advertisement solely to indicate this fact.

${ }^{1}$ Research Plant Pathologist.

${ }^{2}$ Horticulturist.
The use of resistant cultivars is potentially the most economical and reliable method of
×Supplied by M.H. Dickson.

"Supplied by Petoseed, Woodland, Calif. managing this disease. Sources of resistance must, however, be identified for use in plant breeding programs. One -possible source of resistance is the U.S. Plant Introduction collection.

Natti (1958) tested four broccoli and 39 cauliflower plant introductions (PIs) against an isolate of $P$. parasitica that was subsequently designated as race 1 . Natti et al. (1967) tested 230 crucifer PIs for downy mildew resistance. They reported that 16 accessions contained one or more plants resistant to race 2 and, in some cases, race 1 of the pathogen. The former report specified the broccoli and cauliflower PIs that were tested, but the latter report did not specify which crucifer accessions were tested. The purpose of the work reported here was to test all available broccoli and cauliflower accessions in the U.S. Plant Introduction collection for resistance to a local isolate of $P$. parasitica.

The isolate of $P$. parasitica that was used in these tests was obtained in 1987 from fieldgrown plants of B. oleracea cv. 'Calabrese' at Charleston, S.C., and was maintained on cotyledons of the broccoli cultivars Waltham 29 and Green Duke. The techniques described by Williams (1985) were used. The race designation of this isolate was deter-

Table 1. Reaction of Brassica oleracea differential genotypes and control cultivars to a Charleston, S.C., isolate of Peronospora parasitica. Plants inoculated at the two-expanded leaf stage.

\begin{tabular}{|c|c|c|c|c|c|c|c|c|}
\hline \multirow{2}{*}{$\begin{array}{l}\text { Differential } \\
\text { genotype }\end{array}$} & \multirow{2}{*}{$\begin{array}{l}\text { Resistant } \\
\text { to race(s) }\end{array}$} & \multicolumn{6}{|c|}{ Reaction phenotype ${ }^{z}$} & \multirow{2}{*}{$\begin{array}{c}\text { Disease index } \\
\text { (resistance } \\
\text { class) }\end{array}$} \\
\hline & & 0 & 1 & 3 & 5 & 7 & 9 & \\
\hline $\mathrm{B}-\mathrm{O}-23^{\mathrm{x}}$ & 1 & 0 & 0 & 0 & 6 & 26 & 0 & $6.6(S)$ \\
\hline MDR-1w & 1 & 0 & 0 & 0 & 3 & 13 & 0 & $6.6(\mathrm{~S})$ \\
\hline Broccoliw & 1 & 0 & 0 & 0 & 1 & 20 & 0 & 6.9 (S) \\
\hline$M 7-2^{w}$ & 2 & 0 & 16 & 1 & 10 & 0 & 0 & 2.6 (VR) \\
\hline Mll-5w & 2 & 0 & 30 & 3 & 1 & 0 & 0 & $1.3(\mathrm{VR})$ \\
\hline $\mathrm{M} 15 \times \mathrm{M} 24^{\mathrm{w}}$ & 1 and 2 & 0 & 0 & 10 & 11 & 2 & 0 & $4.3(\mathrm{MR})$ \\
\hline MDR $-3^{w}$ & 3 & 0 & 0 & 0 & 7 & 27 & 0 & $6.6(\mathrm{~S})$ \\
\hline $8775-1^{x}$ & 3 & 0 & 0 & 5 & 13 & 24 & 0 & 5.9 (LR) \\
\hline Waltham 29 & None & 0 & 0 & 0 & 7 & 26 & 1 & $6.6(\mathrm{~S})$ \\
\hline Green Duke & None & 0 & 0 & 0 & 2 & 36 & 0 & 6.9 (S) \\
\hline
\end{tabular}

2Reaction classes: 0 = no symptoms; 1 = small necrotic flecks, no sporulation; 3 = necrotic flecks, one to few sporangiophores present; $5=$ necrotic lesions, sparsely scattered sporulation usually confined to necrotic areas; 7 = necrotic lesions, sometimes with accompanying chlorosis, scattered, heavy to abundant sporulation in both chlorotic and necrotic areas; $9=$ necrosis and some chlorosis may be evident, uniformly heavy sporulation over abaxial surface of leaf.

'Resistance classes based on disease indices (DI) calculated by Williams' formula: VR (very resistant) $=\mathrm{DI}$ of $0-3, \mathrm{MR}$ (moderately resistant) $=\mathrm{DI}$ of $3.1-5$; LR (lowly resistant) $=$ DI of 5.1-6; S (susceptible) $=$ DI of 6.1-7; VS (very susceptible) $=$ DI of 7.1-9. 
mined by inoculating it to the $B$. oleracea differentials developed by M.H. Dickson, New York Agricultural Experiment Station, Geneva (Natti et al., 1967; M.H. Dickson, personal communication). The isolate was also used to inoculate broccoli and cauliflower PIs and commercial cultivars to evaluate these accessions for resistance to downy mildew.

Both sets of inoculations and subsequent evaluations of interaction phenotypes were conducted using the following protocol. All work was conducted in glasshouses and growth rooms. A randomized complete-block design with four replicates was used. The pending on availability and viability of seed of each entry. Eight differential genotypes, 240 broccoli and cauliflower PIs, and nine commercial cultivars were tested. Because the growth chamber could not accommodate all of the entries that were tested, the data represent the compilation of four tests. The commercial cultivars Green Duke and Waltham 29 were included in each of these four tests as controls. Data were tested by analysis of variance to determine least significant difference. Based on the reactions of the controls, the tests were homogeneous.

Seed of each entry were sown to Jiffy Mix (Jiffy Products of America, West Chicago) in No. 812 Com-Packs trays (T.O. Plastics, Minneapolis, Minn.). Seedlings were grown in the glasshouse until the cotyledons had fully expanded and were then transferred to a $22 \mathrm{C}$ growth room with a $12-\mathrm{hr}$ photoperiod from sodium vapor lamps. Plants were inoculated when they reached the two-expanded leaf stage. Just before inoculation, the 12 cells of each of the Com-Packs trays were separated to produce four sets of three cells-each. One three-cell set of each of the resultant four sets was randomized and placed in larger plant trays to provide the four replicates.

Inoculum was prepared from the culture of $P$. parasitica maintained on broccoli cotyledons. Cotyledons that supported abundant sporulation by the pathogen were collected and placed in $50 \mathrm{ml}$ of distilled water in a 250-ml flask. The flask was capped and shaken vigorously to dislodge the sporangia. The resulting sporangial suspension was filtered through one layer of cheesecloth and the concentration was determined with the aid of a hemacytometer and adjusted to $5 \times$ $10^{3}$ sporangia per $\mathrm{ml}$. The adaxial surfaces of leaves one and two of all test plants at the three-leaf stage were sprayed with this inoculum to incipient run-off with a Paasche Type-H airbrush (Paasche Airbrush Co., Chicago) at $138 \mathrm{kPa}$. Inoculated plants were placed in a dark\&w chamber at $16 \mathrm{C}$ for 24 chamber and returned to the $22 \mathrm{C}$ growth room. On the 7th day after inoculation, plants were returned to the $16 \mathrm{C}$ dew chamber for $30 \mathrm{hr}$ and ratings of reaction phenotypes were made 9 days postinoculation.

Leaves one and two were observed with the aid of a stereo microscope at magnifications up to $\times 65$ and assigned to the folnumber of plants per replicate varied dehr. Plants were then removed from the dew

Table 2. Reaction of selected U.S. Plant Introductions (PI) and commercial cultivars of broccoli and cauliflower to inoculation with Peronospora parasitica race $2 .{ }^{2}$ PI accessions listed are those in which plants with a resistant reaction phenotype $\leq 3$ were identified.

\begin{tabular}{|c|c|c|c|c|c|c|c|c|c|}
\hline \multirow[b]{2}{*}{ PI/cultivar } & \multicolumn{6}{|c|}{ No: in reaction phenotype ${ }^{y}$} & \multirow{2}{*}{$\begin{array}{l}\text { Total } \\
\text { tested }\end{array}$} & \multicolumn{2}{|c|}{ Disease resistance $^{x}$} \\
\hline & 0 & 1 & 3 & 5 & 7 & 9 & & Index ${ }^{w}$ & Class \\
\hline \multicolumn{10}{|c|}{ Brassica oleracea var. italica (broccoli) } \\
\hline PI 188561 & 0 & 0 & 1 & 8 & 7 & 0 & 16 & 5.8 & LR \\
\hline PI 267721 & 0 & 0 & 1 & 10 & 6 & 0 & 17 & 5.6 & LR \\
\hline PI 462209 & 0 & 0 & 2 & 18 & 12 & 0 & 32 & 5.6 & $\mathrm{LR}$ \\
\hline Green Duke & 0 & 0 & 0 & 15 & 104 & 12 & 131 & 7.0 & $\mathrm{~S}$ \\
\hline Waltham 29 & 0 & 0 & 0 & 3 & 58 & 20 & 81 & 7.4 & VS \\
\hline Emperor & 0 & 0 & 0 & 1 & 26 & 10 & 37 & 7.5 & VS \\
\hline Premium Crop & 0 & 0 & 0 & 0 & 31 & 2 & 33 & 7.1 & VS \\
\hline Green Valiant & 0 & 0 & 0 & 0 & 33 & 5 & 38 & 7.2 & VS \\
\hline Packman & 0 & 0 & 0 & 9 & 26 & 1 & 36 & 6.6 & $\mathrm{~S}$ \\
\hline
\end{tabular}

\section{PI 163485}

PI 165488

PI 173845

PI 181860

PI 183213

PI 183214

PI 188562

PI 204758

PI 204760

PI 204763

PI 204765

PI 204768

PI 204769

PI 204771

PI 204772

PI 204773

PI 204774

PI 204775

PI 204776

PI 204779

PI 204780

PI 208477

PI 208478

PI 208482

PI 208485

PI 226504

PI 231207

PI 231209

PI 241609

PI 241612

PI 261597

PI 264331

PI 264656

PI 264658

PI 267725

PI 291566

PI 291567

PI 343479

PI 345542

PI 373906

PI 373908

PI 373909

PI 373921

PI 385954

PI 385955

PI 390968

PI 419064

PI 462211

PI 462213

PI 462214

PI 462216

PI 462218

PI 462222

PI 462223

PI 462224

PI 462225

PI 478341

Snowball $Y$

Improved

White Rock

Snow Crown 
Table 2. continued

\begin{tabular}{|c|c|c|c|c|c|c|c|c|c|}
\hline \multirow[b]{2}{*}{$\mathrm{PI} /$ cultivar } & \multicolumn{6}{|c|}{ No. in reaction phenotype } & \multirow{2}{*}{$\begin{array}{l}\text { Total } \\
\text { tested }\end{array}$} & \multicolumn{2}{|c|}{ Disease resistance $x$} \\
\hline & 0 & 1 & 3 & 5 & 7 & 9 & & Index ${ }^{w}$ & Class \\
\hline Silver Star & 0 & 0 & 0 & 24 & 5 & 0 & 29 & 5.3 & LR \\
\hline Snow March & 0 & 0 & 0 & 21 & 7 & 0 & 28 & 5.5 & LR \\
\hline
\end{tabular}

Inoculation method is detailed in text.

'Reaction phenotypes: $0=$ no symptoms; $1=$ small necrotic flecks, no sporulation; $3=$ necrotic flecks, one to few sporangiophores present; $5=$ necrotic lesions, sparsely scattered sporulation usually confined to necrotic areas; $7=$ necrotic lesions, sometimes with accompanying chlorosis, scattered, heavy to abundant sporulation in both chlorotic and necrotic areas; $9=$ necrosis and some chlorosis may be evident, uniformly heavy sporulation over abaxial surface of leaf.

×Resistance classes based on disease indices (DI) calculated by Williams' formula: VR (very resistant)

$=$ DI of $0-3, \mathrm{MR}$ (moderately resistant) $=$ DI of $3.1-5$, LR (lowly resistant) $=$ DI of $5.1-6, \mathrm{~S}$ (susceptible) $=$ DI of $6.1-7$, VS (very susceptible) $=$ DI of 7.1-9.

$\mathrm{w}_{\text {LSD }}(P=0.05)$ of \pm 0.9 .

lowing reaction phenotypes: $0=$ no symptoms; 1 = small necrotic flecks, no sporulation; 3 = necrotic flecks, one to few sporangiophores present; $5=$ necrotic lesions, sparsely scattered sporulation usually confined to necrotic areas; 7 = necrotic lesions, sometimes with accompanying chlorosis, scattered, heavy to abundant sporulation in both chlorotic and necrotic areas; $9=$ necrosis and some chlorosis may be evident, uniformly heavy sporulation over abaxial surface of leaf. Using the ratings for reaction phenotype, a disease index (DI) was calculated for each entry by Williams' (1985) formula. Based on these DIs, entries were assigned to the following resistance classes: VR $($ very resistant $)=$ DI of $0-3$; MR $($ mod erately resistant) $=$ DI of 3.1-5; LR (lowly resistant $)=$ DI of 5.1-6; $\mathrm{S}$ ( susceptible $)=$ DI of 6.1-7; VS (very susceptible) = DI of 7.1-9.

The results of the inoculation of the differential genotypes of $B$. oleracea indicated that the test isolate was race 2 of $P$. parasitica (Table 1). The reaction phenotypes and resultant DIs showed that the race 1- and race 3-resistant genotypes were susceptible to this isolate, while the race 2-resistant genotypes were resistant. The genotypes with both race 1 and race 2 resistance exhibited a moderately resistant reaction, which also supports the race 2 designation for the test isolate.

Among the broccoli accessions, PI 267721 and PI 462209, both with DIs of 5.6, were significantly more resistant than any of the commercial broccoli cultivars tested. None of the cauliflower PIs had DIs that indicated they were significantly more resistant than 'Snow Crown', the most resistant cultivar tested $(\mathrm{DI}=4.7)$. Thirteen cauliflower accessions, PIs 181860, 188562, 204765, 204768, 204772, 204773, 204775, 204779, 241612, 264656, 291567, 373906, and 462225, had resistance ratings in the MR class (DI 3.1-5), as did the cultivars Snow Crown and White Rock. However, highly resistant plants that had a reaction phenotype $\leq 3$ were-identified from three broccoli and 57 cauliflower accessions (Table 2). Even though no accessions were identified that had a highly resistant overall DI, the identification of highly resistant plants within many of the tested PI accessions should be useful for the development of resistant cultivars. This list provides plant breeders with a wide range of accessions having a great variety of horticultural characteristics from which to choose.

The resistances that have been used against $P$. parasitica thus far have been conditioned by highly specific major genes (Dixon, 1981), which has limited the usefulness of these resistances because of resistance-breaking races.
Additional testing against other races of downy mildew may indicate that some of the resistances identified in this study are more generalized in nature, which will make them more useful for the control of the disease.

\section{Literature Cited}

Dixon, G.R. 1981. Vegetable crop diseases. Macmillan, London.

Natti, J.J. 1958. Resistance of broccoli and other crucifers to downy mildew. Plant Dis. Rptr. 42:656-662.

Natti, J.J., Dickson, M.H., and J.D. Atkin. 1967. Resistance of Brassica oleracea to downy mildew. Phytopathology 57:144-147.

Sherf, A.F. and A.A. Macnab. 1986. Vegetable diseases and their control. Wiley, New York.

Walker, J.C. and P.H. Williams. 1977. Crucifers, p. 307-325. In: R.R. Nelson (ed.). Breeding plants for disease resistance concepts and applications. The Pennsylvania State Univ. Press, University Park.

Williams, P.H. 1985. Crucifer genetics cooperative resource book. Univ. of Wisconsin, Madison. p. D5-7. 